\title{
A Fiber-Optic Sensor for Leak Detection in a Space Environment
}

\author{
John E. Sinko, ${ }^{*}$ Valentin Korman, ${ }^{\dagger}$ and Adam Hendrickson ${ }^{\ddagger}$ \\ Kratos Defense \& Security Solutions, Madison Research Division, Huntsville, AL 35805 \\ Kurt A. Polzin ${ }^{\S}$ \\ NASA-Marshall Space Flight Center, Huntsville, AL 35812
}

\begin{abstract}
A miniature fiber-optic, laser-based, interferometric leak detector is presented for application as a means to detect on-orbit gas leaks. The sensor employs a fiber-coupled modified Michelson interferometer to detect gas leaks by measuring an increase in gas density in the sensing region. Monitoring changes in the fringe pattern output by the interferometer allows for direct measurement of the gas density in the sensing region and, under the assumption of an equation of state, this can be used to obtain a pressure measurement. Measurements obtained over a pressure range from 20 mtorr to 760 torr using a prototypical interferometer on working gases of air, nitrogen, argon, and helium generally exhibit agreement with a theoretical prediction of the pressure increase required before an interference fringe completely moves over the detector. Additional measurements performed on various gases demonstrate the range of detectible species, measuring sub-torr pressure changes in the process. A high-fidelity measurement places the ultimate pressure resolution for this particular sensor configuration in the $\mathbf{1 0}$ mtorr range. Time-resolved data prove the capability of this sensor to detect fast gas flow phenomena associated with transients and pressure waves.
\end{abstract}

\section{Introduction}

L

ONG-term storage of volatile liquids and high-pressure gases in space is now seen as a necessary step for facilitating safe space travel to celestial objects such as the Moon or Mars. Examples of such mission-critical fluids include cryogenic fuels and oxidizers, high-pressure gases supporting mechanical systems or propulsion, and life-support consumables such as oxygen. Successful storage of such fluids in a space environment is essential for mission success, despite the significant challenges. A leak in a storage or distribution system can cause a variety of problems, including mission-endangering loss of inventory or unbalanced thrust loads on the vehicle. Long-term storage of cryogenic propellants presents a special challenge. The propellant can boil off and be lost through the insulating walls of the tank, or simply escape during thermal cycling of the fittings, valves, and propellant feed lines, when seals are unseated.

Current NASA missions anticipate long-duration in-space storage of propellants, oxidizers, and life support supplies. Leaks of a scale detectable through a pressure drop in the storage tank are often catastrophic and have long been the focus of ground-based mitigation efforts where redundant systems are often employed. However, there is presently no technology available for detecting and monitoring low-level, but still mission-endangering, gas leaks in space. Standard in-space gas detection methods are limited either by their operational pressure range or by selective detection of only certain gases. Mass spectrometer systems are able to perform detection tasks, but their size, mass and use of high voltage, which can potentially cause arcing and ignition of combustible propellants, severely limit their usefulness in space systems.

In this paper, we present a light-based interferometric approach to gas monitoring and leak detection using a fiberoptic, interferometric leak detector sensor. The output of the sensor is the intensity at a point in the interference fringe pattern, which is a function of the gas density (and thus the bulk index of refraction) in the sample region. Changes in the density of gas in the test region cause corresponding changes in the intensity output onto a photodiode detector.

\footnotetext{
${ }^{*}$ Optics Research Scientist.

${ }^{\dagger}$ Senior Optics Research Scientist.

$\ddagger$ Optics Research Scientist. AIAA.

§Propulsion Research Engineer, Propulsion Research and Technology Applications Branch, Propulsion Systems Department. Senior Member

This material is declared a work of the U.S. Government and is not subject to copyright protection in the United States.
} 
This process provides a real-time, temporal history of a leak. The sensor is fiber-coupled and constructed from solid optics, allowing for placement almost anywhere on a spacecraft and for stable, rugged operation, respectively. The sensor consumes very little power (in principle, operation at around $100 \mathrm{~mW}$ input power is realizable), and does not introduce an ignition source.

The outline for the rest of this paper is as follows. In Sect. I, a theoretical treatment of the sensor is presented showing how different gases will affect the output of a sensor. The design of the fiber-coupled sensor tested for this paper is given in Sect. III. The experimental setup used to test the sensor is described in Sect. IV. Finally, testing results are presented in Sect. V.

\section{Sensing Theory}

The interference of two rays of light is a well known physical phenomenon. ${ }^{1}$ Interference is easily understood in the case where the rays each have the same wavelength and there is a phase difference of $\Delta \phi$ between them, as occurs in a 2-path interferometer with a laser light source. For fixed path lengths and for fixed wavelength $\lambda$, the order of interference $m$ can be directly related to the index of refraction $n$ between the reference and signal path lengths. Change in the index of refraction will directly result in a change in the interference order, leading to a fringe shift:

$$
\Delta m=\frac{\Delta \phi}{2 \pi}=\frac{l \Delta n}{\lambda}
$$

where $l$ is the difference between the signal and reference path lengths.

An actual interferometer sensor must include a reference path and a signal path. The beams travelling on these two paths recombine (i.e. interfere) before detection. A longer signal path length will increase $l$ resulting in a higher value of $m$. Since the index of refraction for any gaseous material approaches unity as density is lowered to zero, the sensor must be very sensitive, or it will not be able to observe leaks at low density levels.

The index of refraction $n$ of a medium and the particle density $N$ are related by the Lorentz-Lorenz relation: ${ }^{1}$

$$
N=\frac{3}{4 \pi \alpha} \frac{n^{2}-1}{n^{2}+2}
$$

where $\alpha$ is the bulk polarizability. For an ideal gas, the density may be alternatively expressed as a pressure $P$ :

$$
P=\frac{3}{4 \pi} \frac{R T}{\alpha N_{A}} \frac{n^{2}-1}{n^{2}+2}
$$

where $R$ is the universal gas constant, $T$ is the temperature, and $N_{A}$ is Avagadro's number. The bulk polarizability can be defined in terms of the molar refractivity $A$, typically given in units of $\mathrm{cm}^{3} / \mathrm{mol}$ :

$$
A=\frac{4 \pi}{3} N_{A} \alpha
$$

Values of $A$ are given in the literature for a variety of gases, a few of which are presented in Table 1. A change in the value of the molar refractivity of the test gas will significantly affect the performance of a fiber-optic leak detector sensor. In addition, the sensor should be more sensitive to specific species based on their refractivities. This trait will be discussed in more detail below.

The measurement of interest relies on the ability to discriminate between two pressure values (or density values) by examining the fringe movement; it must relate a change in pressure (density) to the movement of fringes past the photodetector. (Note: thorughout this paper, we refer mostly to changes in pressure since that quantity is more easily measureable by independent means for validation purposes.) By combining Eqs. (1), (3), and (4), assuming near vacuum conditions, $\mathrm{n} \approx 1$, and holding $\lambda, T$, and $l$ constant, an approximate relationship between the time rate of change of pressure and the rate of change in order can be written as:

$$
\frac{\partial P}{\partial t} \approx \frac{2 R T \lambda}{3 A l} \frac{\partial m}{\partial t}
$$

Equation (5) is useful, but the real parameter of interest in evaluating the behavior of the sensor is the rate of change in the pressure with respect to the order of interference (hereafter referred to as $\mu$ ):

$$
\mu \equiv \frac{\partial P}{\partial m} \approx \frac{2 R T \lambda}{3 A l}
$$


Table 1. Molar refractivity values for selected gases.

\begin{tabular}{c|c|c|c}
\hline \hline Gas & Molar Refractivity, $A\left(\mathrm{~cm}^{3} / \mathrm{mol}\right)$ & $T(\mathrm{~K})$ & Source \\
\hline Air & 4.606 & 287.5 & {$[1]$} \\
\hline $\mathrm{Ar}$ & 4.196 & 282.5 & {$[2]$} \\
& $4.1973 \pm 0.0005$ & 298 & {$[3]$} \\
& 4.207 & 299 & {$[4]$} \\
& 4.194 & 298 & {$[5]$} \\
\hline $\mathrm{CO}_{2}$ & 6.642 & 292.6 & {$[2]$} \\
& 6.650 & 299 & {$[4]$} \\
& 6.590 & 298 & {$[5]$} \\
\hline $\mathrm{H}_{2}$ & $2.0845 \pm 0.0004$ & 298 & {$[6]$} \\
\hline $\mathrm{He}$ & 0.5220 & 287.7 & {$[2]$} \\
& $0.5210 \pm 0.0002$ & 303 & {$[7]$} \\
\hline $\mathrm{Kr}$ & 6.377 & 292.6 & {$[2]$} \\
\hline $\mathrm{N}_{2}$ & 4.446 & 292.5 & {$[2]$} \\
& 4.460 & 299 & {$[4]$} \\
& 4.446 & 298 & {$[5]$} \\
& 4.458 & 298 & {$[6]$} \\
& $4.4454 \pm 0.0005$ & 298 & {$[8]$} \\
\hline $\mathrm{Ne}$ & 1.003 & 292.5 & {$[2]$} \\
\hline $\mathrm{O}_{2}$ & 4.028 & 292.5 & {$[2]$} \\
\hline \hline
\end{tabular}

The parameter $\mu$ essentially provides bounds on the limits of sensitivity of the instrument. The integrated result is a linear relationship between the pressure and order. The pressure change per fringe order will decrease with increasing path length and molar refractivity, and increase with increasing temperature and wavelength. In some cases, it may also be useful to consider $\mu^{-1}$. The number of fringes per unit pressure change will directly increase with increasing path length and molar refractivity, and decrease with increasing temperature and wavelength.

The wide range in values of $A$ shown in Table 1 imply that, in principle, it should be possible to detect changes in gas composition within the test region if the gas density (pressure) is otherwise held constant, and the types of gases in the test volume are known. For instance, if the sensor was used to measure pure He instead of pure $\mathrm{CO}_{2}$, a difference of over an order of magnitude would be observed in the value of $\mu$ between the species under the same pressure conditions. A mixture of $\mathrm{He}$ and $\mathrm{CO}_{2}$ would have a mean polarizability somewhere between that of a pure sample of $\mathrm{He}$ or $\mathrm{CO}_{2}$, leading to a molar refractivity bounded by the values for the two species. Given that the species and pressure are known, the intensity of the observed interference fringe would allow for an exact measurement of the ratio of the two gas species present.

\section{Sensor Design}

The sensor design was aimed at producing a detector that was compact, robust, and sensitive. A longer sensing path length leads to increased sensitivity, but it also increases the overall size of the device. Single mode fiber was used for delivery of the laser light to the sensor. Use of single mode fiber eliminated internal fiber interference between different wave propagation modes, resulting in a sensor output that varied smoothly and uniformly as the interference fringes moved relative to the return (sensor output) fiber optic. Simple light interference was used in this case, as opposed to the more complex light beam frequency modulation techniques common to other interferometric sensors.

A photograph and schematic of a prototypical lab-model sensor are shown in Fig. 1. In the interferometer, light follows two major paths; a reference path and a sensing path. Light enters through one of the fiber optic cables at right, is collimated by a gradient index (GRIN) lens, and is split by the first beamsplitter. The reference path light travels within the two beamsplitters, while the sensing path light transitions to a free beam and passes through a volume of 
gas. After reflecting off two mirrors, the sensing beam returns to the second beamsplitter where it recombines with the reference beam, producing an interference pattern. The light is focused by a second GRIN lens into a fiber optic cable that leads to a photodiode detector. Changes in the gas within the sensing path region cause the interference pattern produced by the combination of the sensing and reference beams to move, and this motion is detected by the photodiode and correlated into a measurement of gas density (or pressure). For simplicity, the entire sensor was constructed on a rectangular ground glass BK7 base plate.

\section{Experimental Setup}

Testing was conducted in the small stainless steel vacuum chamber shown schematically in Fig. 2, which measures approximately $30 \mathrm{~cm}$ in diameter and $1 \mathrm{~m}$ in length. The chamber was evacuated to a $10^{-3}$ torr base for the experiments presented in this paper. Light for the sensor is conducted into and out of the chamber using a pair of sealed fiber optic vacuum feedthroughs. The light signal is generated using a $632.8 \mathrm{~nm} \mathrm{CW} \mathrm{HeNe}$ laser with a beam power of $30 \mathrm{~mW}$. In testing, the operation of the sensor was found to be roughly independent of the polarization of the laser beam.

(A)

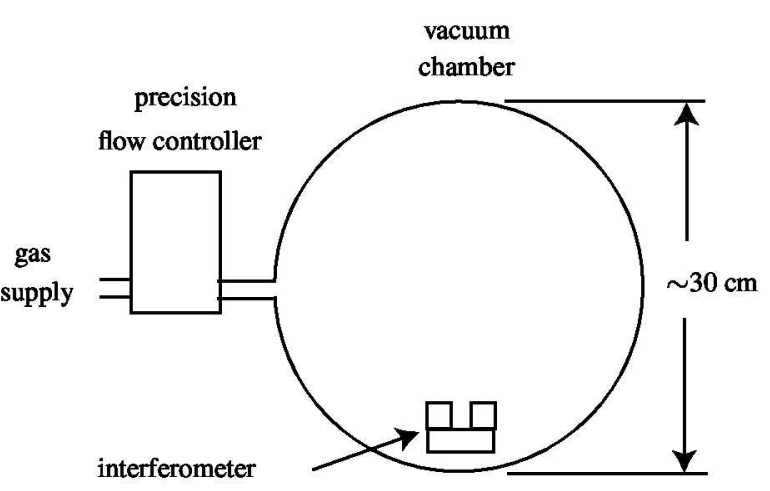

(B)

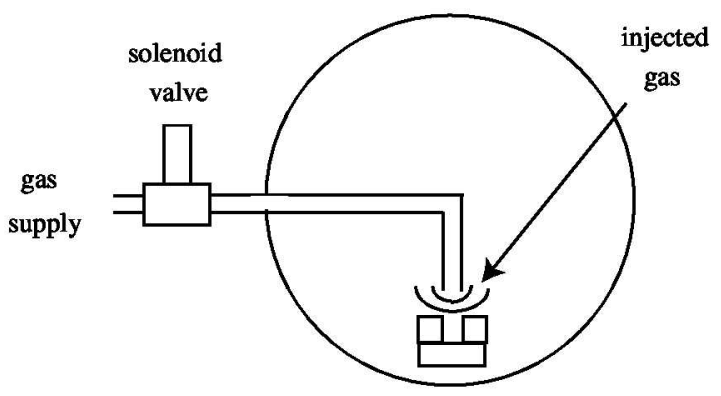

Figure 2. Schematic of the experimental apparatus used for (A) controlled gas injection testing and (B) fast, pulsed gas injection testing.

alignment of the interferometer, these peaks should repeat and be indistinguishable from each other. parameters. gas density rise time in the sensing region.

\section{Results}

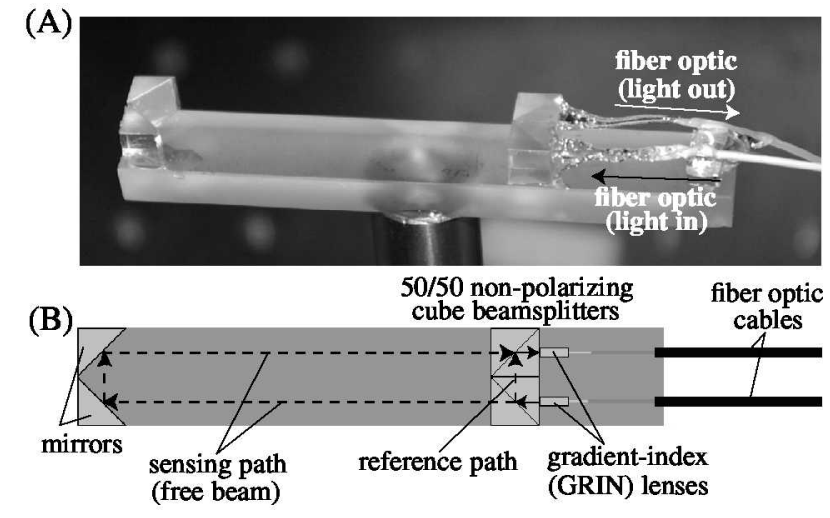

Figure 1. (A) Photograph and (B) schematic of a fiber-coupled gas detection sensor.

Controlled gas injection was performed using an MKS 1479 precision flow controller. Testing was conducted by isolating the chamber from its vacuum pumping system and then introducing gas into the chamber at a specified volumetric flow rate. The signal from the sensor was measured using a silicon photodiode. An independent measure of the gas pressure in the chamber was performed using an MKS baratron gauge, which is a gas-independent capacitance manometer pressure gauge. Both the photodiode and baratron voltage outputs were simultaneously recorded to yield a time history of both

Fast gas injection was performed using a solenoid valve, which was energized for almost a second before being closed. The valve was fed by air at atmospheric pressure, and the flow was directed by a tube with the exit placed just above the sensor. This was done to maximize the gas density at the sensor while minimizing the

The data presented in this section serve to provide an illustrative representation of the capabilities of the sensor in its present form. Notionally, we expect that as the density (or alternatively the pressure) increases, changing the index of refraction of the gas in the sensing path, interference fringes will pass by the detector resulting in the intensity peaks and valleys shown in Fig. 3. Assuming constant temperature and perfect rigidity in the 


\section{A. Controlled Gas Injection}

Measurements performed to validate the response of the sensor as a function of increasing gas pressure are presented in Fig. 4. These data were obtained using the configuration shown in Fig. 2A. This configuration provided a controllable source of the gas flow rate into the vacuum chamber. Pressure measurements were obtained to correlate the increase in pressure with the number of fringes passing the detector. In all cases, the flow rate was set to $1500 \mathrm{sccm}$. Gases represented in Fig. 4A-D were air, nitrogen, argon, and helium, respectively.

The fringe spacings for air, nitrogen, and argon were 26.4, 26.5, and 28.4 torr/fringe, respectively. These data compare favorably with Eq. (6) when the actual sense path length, laser wavelength, and the values of molar refractivity for each gas (Table 1) are used to compute

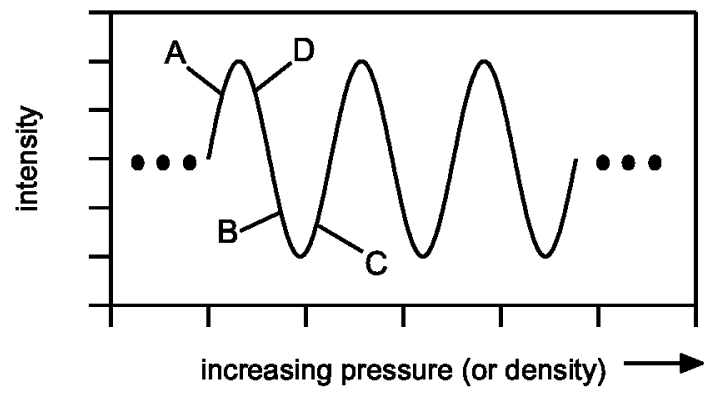

Figure 3. Plot showing the notional response of the interferometer as a function of increasing pressure and/or density (at constant temperature).

the number of torr per fringe. The data for He is different from the other three sets in two ways. First, there are far fewer fringes over the pressure range tested, which is expected since the molar refractivity is much smaller in this case than for the other three gases tested. Also, the fringe spacing as a function of pressure was not observed to be uniform. This could be an effect of the residual gas that remained in the vacuum chamber after evacuation. At the lower pressure levels the measurement is effectively being performed on a mixture comprised of air and helium, with a molar refractivity of the mixture somewhere between the values for either gas. As more He was injected into the chamber, the response of the system is governed more by the molar refractivity value of pure He, and the fringe spacing as a function of pressure becomes more uniform. Since air, argon, and nitrogen all have similar values of $A$, this effect did not appear in any noticeable fashion in those data sets.
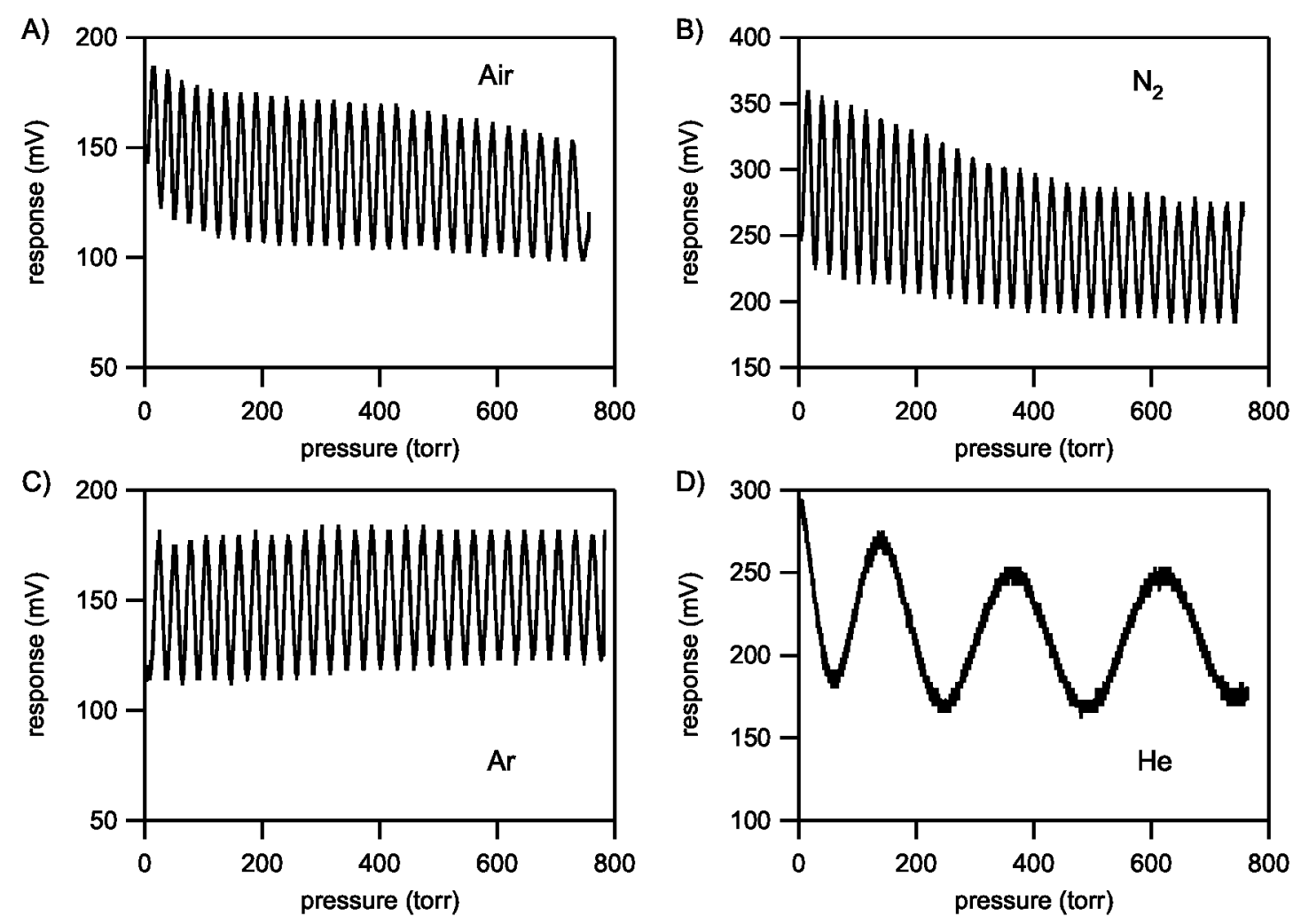

Figure 4. Interferometer measured response to moving interference fringes as a function of pressure (from 0-760 torr) for A) air, B) nitrogen, C) argon, and D) helium. 
Additional data are presented in Fig. 5 for air, argon, carbon dioxide, and krypton, showing the interferometer response as a function of increasing pressure, displayed on a semilog scale from a rough vacuum level of $\sim 100$ mtorr up to about 10 torr. These data we acquired at a flow rate of $35 \mathrm{sccm}$ to allow for better resolution at the low end of the pressure regime. The difficulty in generating and analyzing these data using the present setup is that the interferometer does not always start at the same point in the interference pattern, so it may be deeper in a valley or nearer to a peak for different trials. However, these data displayed on the semilog axis show the pressure level where the sensor is effective in detecting changes. At these moderate flow rates and for the prototypical sensor used in this study, this sensitivity is well below 1 torr.

\section{B. Pressure Resolution}

The data in Fig. 6 show the response of the sensor as air slowly leaked into the vacuum chamber, increasing the pressure over $100 \mathrm{~s}$ from $20 \mathrm{mtorr}$ to $30 \mathrm{mtorr}$. While the relative noise in this particular data set is quite large (roughly $40 \mathrm{mV}$ peak-to-peak) the sensor output exhibits a general trending in the positive direction as the pressure increases, serving to demonstrate a pressure resolution on air below $10 \mathrm{mtorr}$. With a cleaner voltage signal from the photodiode, greater magnification on the fringe, or an increase in the path length $l$, it is expected that resolutions several orders of magnitude lower than 10 mtorr can be achieved.

\section{Fast Gas Injection}

Data obtained from fast gas injection testing performed using the configuration shown in Fig. 2B are presented in Fig. 7A-D for similar gas pulses at acquisition rates of $10,50,250$, and $500 \mathrm{ksamples} / \mathrm{sec}$, respectively. The solenoid valve is opened at time $t=0$, and closed less than a second later. For these tests, the tank pressure started at $\sim 10 \mathrm{mtorr}$ and ended at 5-10 torr.

In Fig. 7A, the signal from the interferometer first rises quickly, then falls to a lower level, continuing to decrease at a slow rate after the initial transient before reversing and beginning to rise again just after $500 \mathrm{msec}$. When the valve is closed, there is a slight dip in the signal before it quickly increases back to roughly the initial output level. This pattern can be understood using the notional plot in Fig. 3. In the notional plot, the interferometer's initial output is represented by point $\mathrm{A}$ on the curve. As the solenoid valve is energized, the signal quickly rises to the peak level before dropping to the level of point B on the curve. Over the next $800 \mathrm{msec}$, as the choked flow set up a quasi-steady flowfield with a slightly increasing density in the interferometer sense region, the signal continues to decrease through a valley,
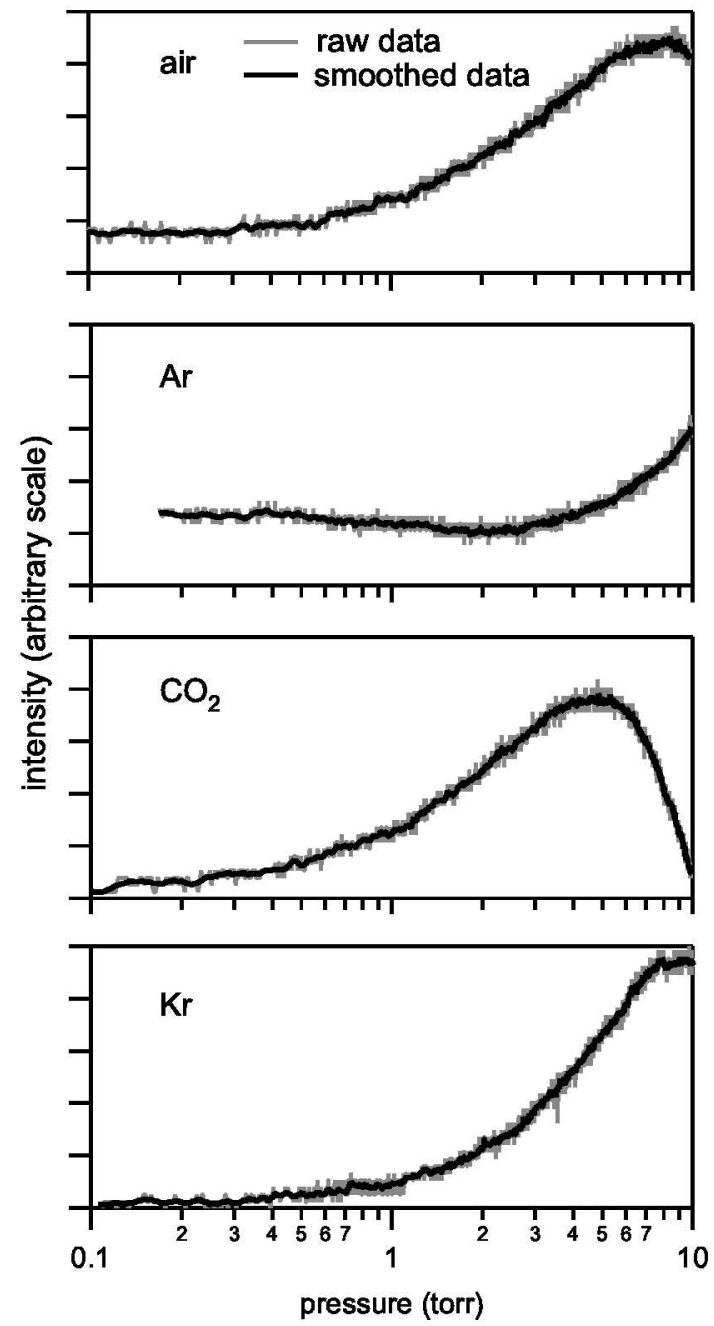

Figure 5. Interferometer measured response to moving interference fringes as a function of pressure (at low pressure levels displayed on a semilog scale) for air, argon, carbon dioxide, and krypton. The intensity scale spans the same range for all plots.

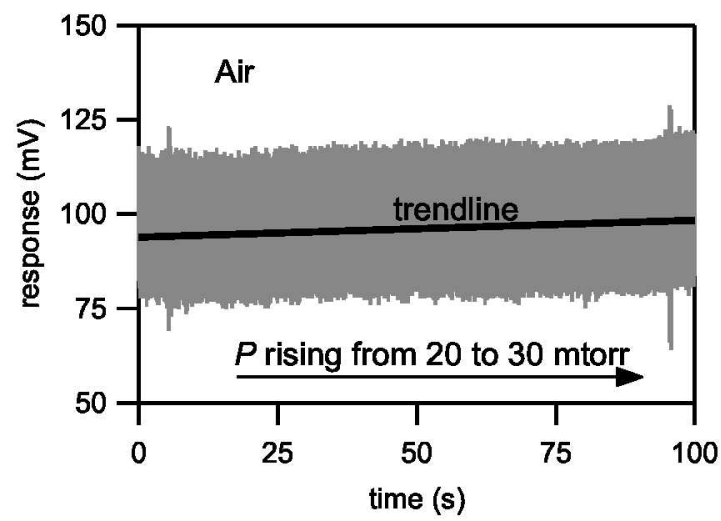

Figure 6. Interferometer response as the vacuum chamber pressure rose from 20 to $30 \mathrm{mtorr}$ due to a slow leak in the vacuum system. 6 of 8 

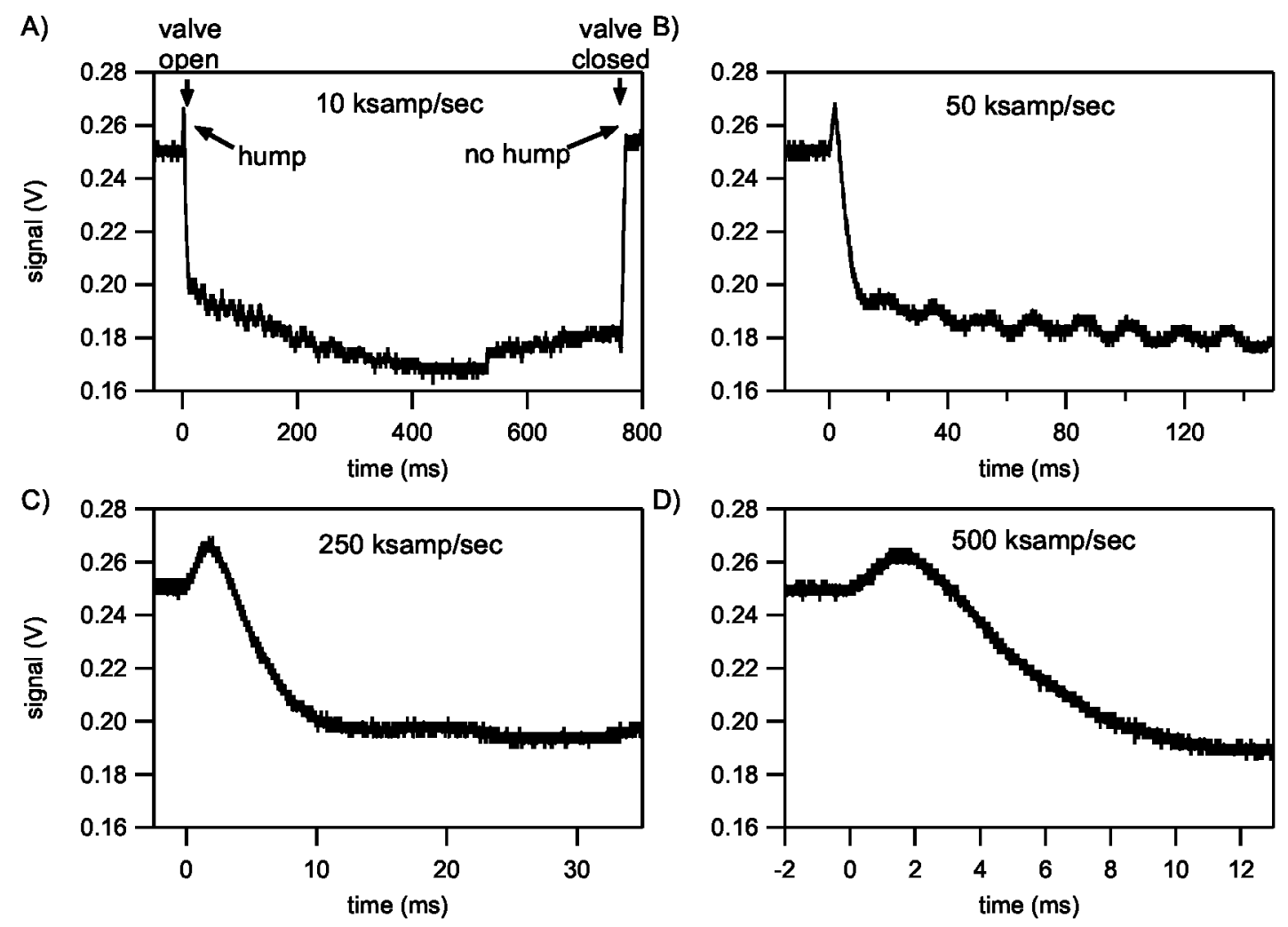

Figure 7. Interferometer response to a short, fast gas pulse injected at the sensor head acquired at A) $10 \mathrm{ksamp} / \mathrm{sec}$, B) $50 \mathrm{ksamp} / \mathrm{sec}$, C) $250 \mathrm{ksamp} / \mathrm{sec}$ and D)500 ksamp/sec.

reaching point $\mathrm{C}$ as the valve is closed. When the valve is closed, interrupting the gas source, the local density at the interferometer decreases again, and the output of the sensor travels backwards from $C$ through the interference fringe minimum and up to D. The data in Fig. 7B-D demonstrate that the sensor has the capability for time-resolved measurements on the order of the response time of the photodiode being employed. In addition, these measurements show that the initial transient behaves in the manner described above.

Another noteworthy feature in the pulsed gas injection data is observed in Figs. 7A-B. At $10 \mathrm{ksamples} / \mathrm{sec}$, after the initial transient there is considerable hash in the data, which could be taken to represent noise. However, at 50 ksamples/sec, we observe that there is actually a regular, repeating oscillation superimposed on the main signal. The first oscillation and a half appear in the data presented in Fig. 7C and correlate with the commensurate data in Fig. 7B. These oscillations do not correlate with any AC electrical signal $(60 \mathrm{~Hz})$ and have more structure to them than experimental 'noise', implying that this response is caused by the detection of compression and rarefaction waves passing through the sensing region of the interferometer.

\section{Conclusions}

On-orbit sensing of gas leaks in mission-critical components and systems can assist in reducing risk and enhancing the overall safety, increasing the probability of mission success. A fiber optic-coupled interferometric leak detection sensor has advantages over other state-of-the-art detection techniques, making it well suited for deployment in space. The fiber-coupled nature of the sensor allows for remote placement near critical areas on a spacecraft. In addition, the sensor is compact, relatively unaffected by many environmental factors, and does not introduce an ignition source into what could be an explosive fuel/oxidizer mixture. Measurements obtained using a prototypical interferometric sensor generally agree favorably with theoretical predictions of the number of torr per fringe over a pressure range from 20 mtorr to 760 torr. Deviations from the theoretical response for He, especially in the lower pressure regime, can be understood as being a measurement of a gas mixture comprised of residual air in the test vessel and injected He. Plotting the sensor response as a function of the pressure (on a semilog scale) demonstrates a sensitivity of under 
1 torr for most of the gases tested. In a very fine resolution test, the sensor demonstrated a pressure resolution on air below $10 \mathrm{mtorr}$. Pulsed gas-injection data show the ability to use the sensor to acquire fast, time-resolved transient data, capturing not only the bulk pressure variations but also much faster flow ocsillation phenomena.

\section{Acknowledgments}

We are grateful for the project support provided by the NASA-MSFC Technology Transfer Office. Funding for MRD Kratos was provided under NASA contract NAS8-02060. We gratefully acknowledge the MSFC support staff of Tommy Reid, Doug Galloway, and Roger Harper and undergraduate students Adam Kimberlin, Joe Balla, and Jarred Reneau for their contributions to this effort. We also thank Ben Ramsey, Randy James, and Jason Hughes of MRD Kratos for their assistance in the fabrication of components used in this study. This work benefitted from several insightful suggestions and comments made by Prof. Don Gregory of the University of Alabama in Huntsville and Dr. Kenneth Herren of MSFC. Finally, we want to thank Mr. James Martin and Mr. J. Boise Pearson for continued management support of this effort.

\section{References}

${ }^{1}$ M. Born and E. Wolf, Principles of Optics, 7th expanded edition, Cambridge University Press, Cambridge, 1999.

${ }^{2}$ K.P. Birch, "Precise determination of refractometric parameters for atmospheric gases," J. Opt. Soc. Am. A, 8(4):647, 1991

${ }^{3}$ R. Coulon, G. Montixi, and R. Occelli, "Determination experimentale des coefficients du viriel de la refractivite des gaz : etude de l'argon (Experimental determination of the refractivity viral coefficients for argon gas)," Can. J. Phys., 59(10):1555, 1981.

${ }^{4}$ A.D. Buckingham and C. Graham, "The density dependence of the refractivity of gases," Proc. R. Soc. A, 336:275, 1974.

${ }^{5}$ R.C. Burns, C. Graham, and A.R.M. Weller, "Direct measurement and calculation of the second refractivity virial coefficients of gases," Mol. Phys., 59(1):41, 1986.

${ }^{6}$ D.E. Diller, "Refractive index of gaseous and liquid hydrogen," J. Chem. Phys., 49(7):3096, 1968.

${ }^{7}$ S. Kirouac and T.K. Bose, "Polarizability and dielectric properties of helium," J. Chem. Phys., 64(4):1580, 1976.

${ }^{8} \mathrm{G}$. Montixi, R. Coulon, and R. Occelli, "Coefficients du viriel de la refractivite de l'azote a $25^{\circ} \mathrm{C}$ (Refractivity virial coefficients for nitrogen at $\left.25^{\circ} \mathrm{C}\right), "$ Can. J. Phys., 61(3):473, 1983. 